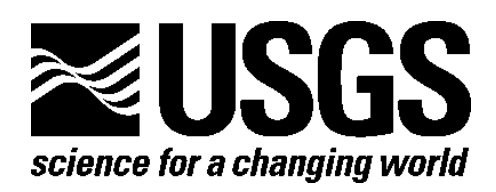

\title{
Sea Surface Temperature Estimates for the Mid- Piacenzian Indian Ocean-Ocean Drilling Program Sites 709, 716, 722, 754, 757, 758, and 763
}

By Marci M. Robinson, Harry J. Dowsett, and Danielle K. Stoll

Open-File Report 2017-1158

U.S. Department of the Interior U.S. Geological Survey 


\section{U.S. Department of the Interior \\ Ryan K. Zinke, Secretary}

\section{U.S. Geological Survey \\ William H. Werkheiser, Deputy Director \\ exercising the authority of the Director}

U.S. Geological Survey, Reston, Virginia: 2018

For more information on the USGS-the Federal source for science about the Earth,

its natural and living resources, natural hazards, and the environment-visit

https://www.usgs.gov/ or call 1-888-ASK-USGS (1-888-275-8747).

For an overview of USGS information products, including maps, imagery, and publications, visit https://store.usgs.gov.

Any use of trade, firm, or product names is for descriptive purposes only and does not imply endorsement by the U.S. Government.

Although this information product, for the most part, is in the public domain, it also may contain copyrighted materials as noted in the text. Permission to reproduce copyrighted items must be secured from the copyright owner.

Suggested citation:

Robinson, M.M., Dowsett, H.J., and Stoll, D.K., 2018, Sea surface temperature estimates for the midPiacenzian Indian Ocean-Ocean Drilling Program sites 709, 716, 722, 754, 757, 758, and 763: U.S. Geological Survey Open-File Report 2017-1158, 14 p., https://doi.org/10.3133/ofr20171158.

ISSN 2331-1258 (online) 


\section{Contents}

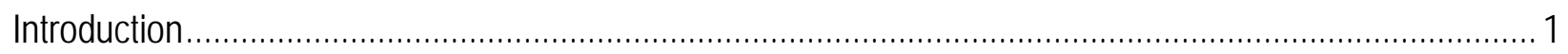

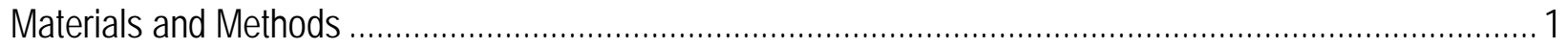

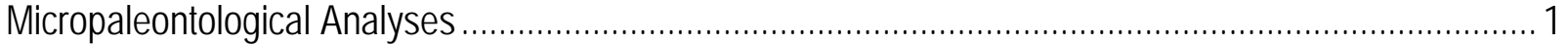

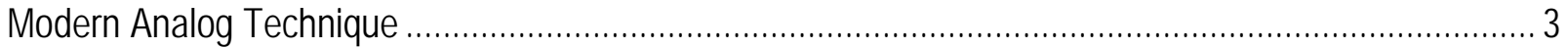

Factor Analytic Transfer Function............................................................................................. 4

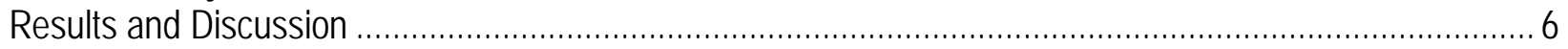

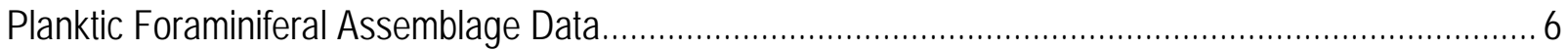

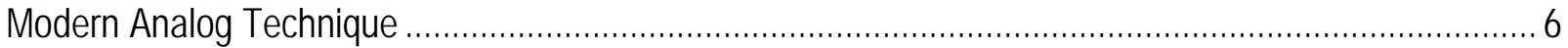

Factor Analytic Transfer Function.......................................................................................

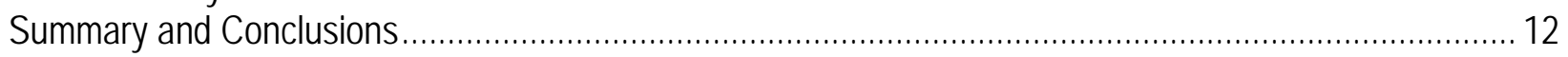

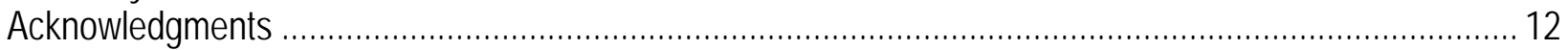

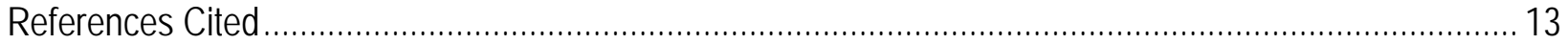

\section{Figures}

1. Map showing locations of Ocean Drilling Program sites $709,716,722,754,757,758$, and 763 described in this study and mean mid-Piacenzian planktic foraminifer species distribution at

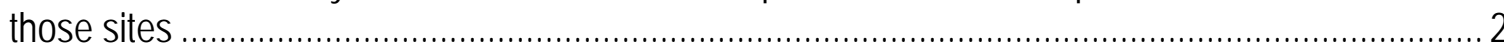

2. Time series plots of mid-Piacenzian planktic foraminifer species assemblages by percentage for Ocean Drilling Program sites 709, 716, 722, 754, 757, 758, and 763 in the Indian Ocean, arranged geographically ................................................................................................ 6

3. Downcore variation in factor loadings for F1 (subtropical), F2 (boundary current), F3 (tropical), F4 (transitional), and F5 (polar-subpolar) and downcore variation in communality (dashed line) for Ocean Drilling Program sites 709, 716, 722, 754, 758, and 763.

\section{Tables}

1. Site information for Ocean Drilling Program sites $709,716,722,754,757,758$, and 763

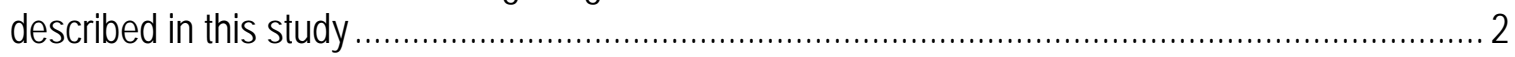

2. Taxonomic counting categories used in this study ................................................................. 3

3. Varimax assemblage description matrix showing the relative importance of each of the 33 variables to each of the five primary factors

4. Cold and warm season sea surface temperature estimates for samples using the modern analog technique with squared chord distance values and the factor analytic transfer function GSF36 with communalities 


\section{Conversion Factors}

International System of Units to U.S. customary units

\begin{tabular}{|c|c|c|}
\hline Multiply & By & To obtain \\
\hline \multicolumn{3}{|c|}{ Length } \\
\hline centimeter $(\mathrm{cm})$ & 0.3937 & inch (in.) \\
\hline millimeter (mm) & 0.03937 & inch (in.) \\
\hline meter $(\mathrm{m})$ & 3.281 & foot $(\mathrm{ft})$ \\
\hline meter $(\mathrm{m})$ & 1.094 & yard (yd) \\
\hline \multicolumn{3}{|c|}{ Volume } \\
\hline liter (L) & 33.81402 & ounce, fluid (fl. oz) \\
\hline liter (L) & 2.113 & pint (pt) \\
\hline liter (L) & 1.057 & quart (qt) \\
\hline liter (L) & 0.2642 & gallon (gal) \\
\hline cubic meter $\left(\mathrm{m}^{3}\right)$ & 264.2 & gallon (gal) \\
\hline cubic decimeter $\left(\mathrm{dm}^{3}\right)$ & 0.2642 & gallon (gal) \\
\hline cubic centimeter $\left(\mathrm{cm}^{3}\right)$ & 0.06102 & cubic inch (in ${ }^{3}$ ) \\
\hline liter (L) & 61.02 & cubic inch (in $\left.{ }^{3}\right)$ \\
\hline \multicolumn{3}{|c|}{ Mass } \\
\hline gram (g) & 0.03527 & ounce, avoirdupois (oz) \\
\hline \multicolumn{3}{|c|}{ Density } \\
\hline gram per cubic centimeter $\left(\mathrm{g} / \mathrm{cm}^{3}\right)$ & 62.4220 & pound per cubic foot $\left(\mathrm{lb} / \mathrm{ft}^{3}\right)$ \\
\hline
\end{tabular}

Temperature in degrees Celsius $\left({ }^{\circ} \mathrm{C}\right)$ may be converted to degrees Fahrenheit $\left({ }^{\circ} \mathrm{F}\right)$ as ${ }^{\circ} \mathrm{F}=\left(1.8 \times{ }^{\circ} \mathrm{C}\right)+32$.

Temperature in degrees Fahrenheit $\left({ }^{\circ} \mathrm{F}\right)$ may be converted to degrees Celsius $\left({ }^{\circ} \mathrm{C}\right)$ as ${ }^{\circ} \mathrm{C}=\left({ }^{\circ} \mathrm{F}-32\right) / 1.8$.

\section{Abbreviations}

$\begin{array}{ll}\text { BFD } & \text { Brown University Foraminiferal Database } \\ \text { IODP } & \text { International Ocean Discovery Program } \\ \text { MAT } & \text { modern analog technique } \\ \text { NCEI } & \text { National Centers for Environmental Information } \\ \text { ODP } & \text { Ocean Drilling Program } \\ \text { PRISM } & \text { Pliocene Research, Interpretation and Synoptic Mapping } \\ \text { SCD } & \text { squared chord distance } \\ \text { SST } & \text { sea surface temperature } \\ \text { USGS } & \text { U.S. Geological Survey }\end{array}$




\title{
Sea Surface Temperature Estimates for the Mid- Piacenzian Indian Ocean-Ocean Drilling Program Sites 709, 716, 722, 754, 757, 758, and 763
}

\author{
By Marci M. Robinson, Harry J. Dowsett, and Danielle K. Stoll
}

\section{Introduction}

Despite the wealth of global paleoclimate data available for the warm period in the middle of the Piacenzian Stage of the Pliocene Epoch (about 3.3 to 3.0 million years ago [Ma]; Dowsett and others, 2013, and references therein), the Indian Ocean has remained a region of sparse geographic coverage in terms of microfossil analysis. In an effort to characterize the surface Indian Ocean during this interval, we examined the planktic foraminifera from Ocean Drilling Program (ODP) sites 709, 716, 722, 754, 757, 758, and 763, encompassing a wide range of oceanographic conditions. We quantitatively analyzed the data for sea surface temperature (SST) estimation using both the modern analog technique (MAT) and a factor analytic transfer function. The data will contribute to the U.S. Geological Survey (USGS) Pliocene Research, Interpretation and Synoptic Mapping (PRISM) Project's global SST reconstruction and climate model SST boundary condition for the mid-Piacenzian and will become part of the PRISM verification dataset designed to ground-truth Pliocene climate model simulations (Dowsett and others, 2013).

\section{Materials and Methods}

ODP sites 709, 716, 722, 754, 757, 758, and 763 cover the Indian Ocean north of $40^{\circ} \mathrm{S}$., with average spacing between sites of less than $10^{\circ}$ in both latitude and longitude (fig. 1 , table 1). We focused our analysis on the warm interval of the mid-Piacenzian as described in Dowsett and others (2013). Age models used to determine the age of samples at each site are listed in table 1.

\section{Micropaleontological Analyses}

Mid-Piacenzian core material was sampled for quantitative planktic foraminiferal faunal analyses. Samples of 20 cubic centimeters $\left(\mathrm{cm}^{3}\right)$ from site 754 , originally collected for ostracod trace metal analysis, and $10 \mathrm{~cm}^{3}$ from all other sites were oven dried at $\leq 50$ degrees Celsius $\left({ }^{\circ} \mathrm{C}\right)$. Dried bulk samples were disaggregated in 250 milliliters (ml) of warm tap water with about $50 \mathrm{ml}$ of dilute sodium hexametaphosphate (5 grams per liter water). Samples were then agitated for 1 hour at about $21^{\circ} \mathrm{C}$ and washed over a 150 -micrometer sieve using a fine spray hose. The coarse fraction was dried in an oven at $\leq 50{ }^{\circ} \mathrm{C}$. Some samples required an additional wash in order to obtain clean specimens. The sample was split using a precision microsplitter to obtain 300 to 350 specimens for analysis. Most samples required 8 to 10 splits to achieve 300 to 350 
specimens. Foraminifera were least abundant at sites 722 and 758 where one-half to all of the specimens were picked for analysis. Individual specimens were identified to species level, generally following the taxonomy of Parker $(1962,1967)$ and Blow (1969) with modifications (Dowsett and Robinson, 2007), and glued to a 60-square micropaleontological slide.

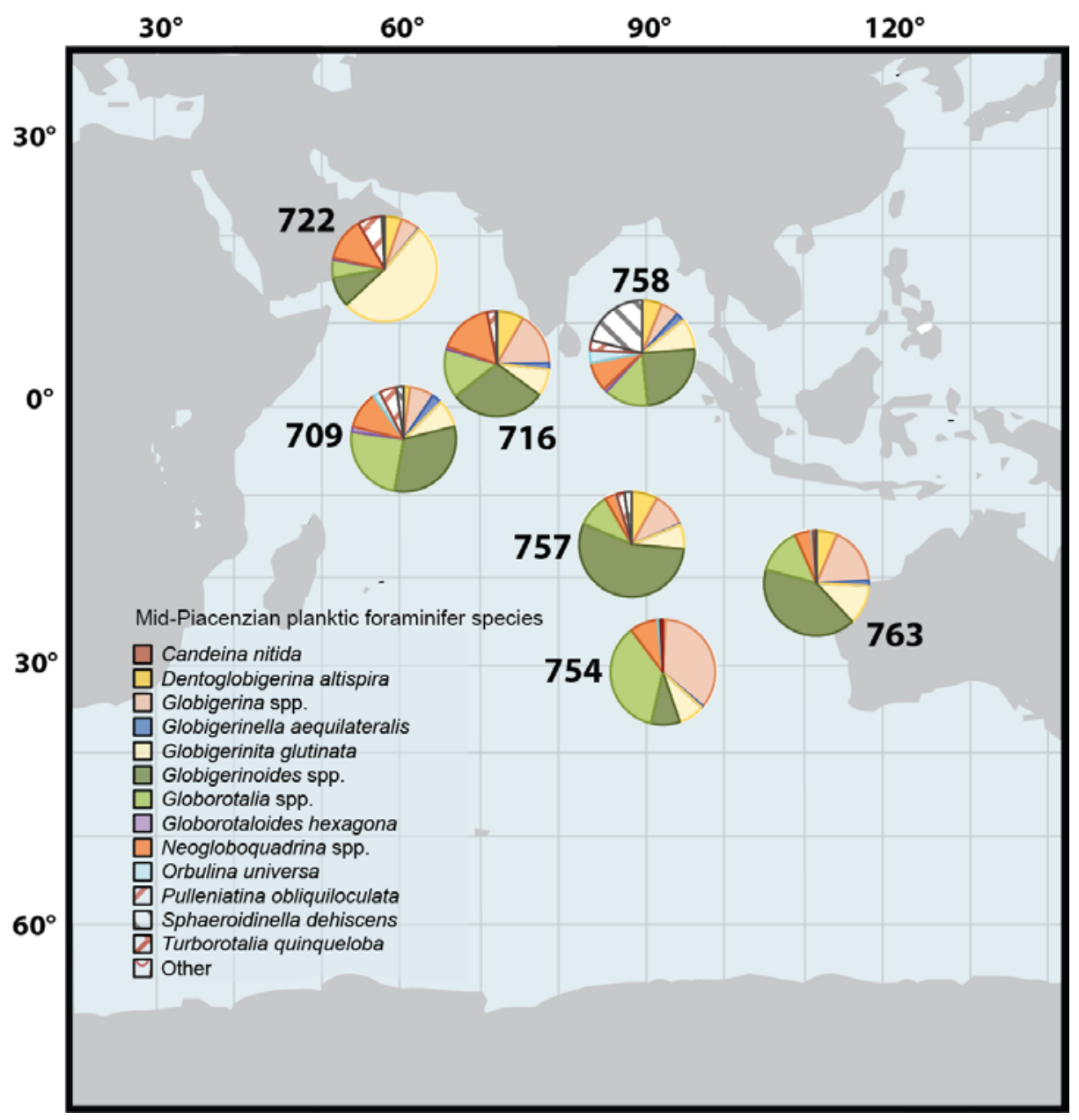

Figure 1. Map showing locations of Ocean Drilling Program sites 709, 716, 722, 754, 757, 758, and 763 described in this study and mean mid-Piacenzian planktic foraminifer species distribution at those sites.

Table 1. Site information for Ocean Drilling Program sites 709, 716, 722, 754, 757, 758, and 763 described in this study.

[ODP, Ocean Drilling Program; m, meter]

\begin{tabular}{crrrl}
\hline ODP site & Latitude & Longitude & Water depth $(\mathrm{m})$ & \multicolumn{1}{c}{ Age model reference } \\
\hline 709 & $3.92^{\circ} \mathrm{S}$. & $60.55^{\circ} \mathrm{E}$. & 3,038 & Karas and others, 2011 \\
716 & $4.93^{\circ} \mathrm{N}$. & $73.28^{\circ} \mathrm{E}$. & 544 & Rio and others, 1990 \\
722 & $16.62^{\circ} \mathrm{N}$. & $59.80^{\circ} \mathrm{E}$. & 2,028 & Herbert and others, 2010 \\
754 & $30.93^{\circ} \mathrm{S}$. & $93.57^{\circ} \mathrm{E}$. & 1,064 & Shipboard Scientific Party, 1989a \\
757 & $17.02^{\circ} \mathrm{S}$. & $88.18^{\circ} \mathrm{E}$. & 1,652 & Shipboard Scientific Party, 1989b \\
758 & $5.38^{\circ} \mathrm{N}$. & $90.37^{\circ} \mathrm{E}$. & 2,924 & Farrell and others, 1995 \\
763 & $20.59^{\circ} \mathrm{S}$. & $112.21^{\circ} \mathrm{E}$. & 1,368 & Karas and others, 2011 \\
\hline
\end{tabular}




\section{Modern Analog Technique}

The modern analog technique quantifies faunal changes within deep sea cores in terms of modern oceanographic conditions (Hutson, 1980). The method uses a measure of faunal dissimilarity to compare downcore samples to each reference sample in a modern faunal database. We chose the following squared chord distance (SCD) measure for our study:

$$
d_{i j}=\Sigma_{k}\left(p_{i k}^{0.5}-p_{j k}^{0.5}\right)^{2}
$$

where $d_{i j}$ is the squared chord distance between two multivariate samples $i$ and $j, p_{i k}$ is the proportion of species $k$ in sample $i$, and $p_{j k}$ is the proportion of species $k$ in sample $j$. Squared chord distance values can range from 0.0 to 2.0, with 0.0 indicating identical proportions of species in the samples being compared.

Table 2. Taxonomic counting categories used in this study.

\begin{tabular}{lc}
\multicolumn{1}{c}{ Taxon } & $\begin{array}{c}\text { Maximum occurrence } \\
\text { (percent of sample) }\end{array}$ \\
\hline Globigerinoides ruber & 57.7 \\
Globigerinoides sacculifer & 24.8 \\
Globigerinita glutinata & 41.7 \\
Globigerinella aequilateralis & 9.1 \\
Neogloboquadrina dutertrei & 35.0 \\
Globigerina calida & 8.8 \\
Globigerina bulloides & 62.2 \\
Pulleniatina obliquiloculata & 20.8 \\
Globigerinoides conglobatus & 8.1 \\
Globorotalia truncatulinoides (dextral) & 10.5 \\
Globigerina falconensis & 19.5 \\
Globorotaloides hexagona & 8.0 \\
Globigerinoides tennellus & 7.7 \\
Globigerina rubescens & 5.2 \\
Orbulina universa & 4.5 \\
Globorotalia truncatulinoides (sinistral) & 12.9 \\
Beella digitata & 12.7 \\
Neogloboquadrina pachyderma (sinistral) & 97.5 \\
Globorotalia crassaformis & 4.4 \\
Candeina nitida & 1.7 \\
Globorotalia menardii flexuosa & 2.2 \\
Globorotalia hirsuta & 4.4 \\
Sphaeroidinella dehiscens & 5.6 \\
Turborotalita humilis & 1.1 \\
Turborotalita quinqueloba & 16.5 \\
Hastigerina adamsi & 0.7 \\
Neogloboquadrina “du-pac” & 27.0 \\
Globorotalia tumida & 23.5 \\
Globorotalia menardii & 65.8 \\
Neogloboquadrina pachyderma (dextral) & 29.4 \\
Globorotalia inflata & 83.6 \\
\hline
\end{tabular}

Census data from the mid-Piacenzian Indian Ocean samples were compared to census data from 279 modern core-top Indian Ocean samples of the Brown University Foraminiferal Database (BFD) surface dataset (Prell and others, 1999). We revised the modern planktic foraminifer data into categories that retained most of the primary temperature signal but also 
provided a good fit to mid-Piacenzian assemblages. The modern taxa were reduced to 33 midPiacenzian counting categories by combining taxa that have documented similar environmental tolerances (table 2), following Dowsett and Poore (1990) and Dowsett (1991). Modern cold and warm season SSTs at all core locations were calibrated to the Reynolds and Smith (1995) modern surface temperature dataset (see Dowsett and Poore, 1990; Dowsett, 1991).

\section{Factor Analytic Transfer Function}

Census data from the same BFD samples as used in the MAT were used to construct a factor analytic transfer function to document and compare the spatial distribution of modern and Pliocene taxa. The modern taxa, regrouped as for the MAT, were quantitatively analyzed using a Q-mode factor analysis approach in order to determine interrelationships between samples. Our analysis identified five primary factors (F1-F5) that accounted for $92 \%$ of the total variance in the dataset (table 3). The relative importance of each of the 33 counting categories (used here as factor analytic variables) to each factor is presented in the factor score matrix. The counting categories with the greatest contribution to the F1 assemblage are Globigerinoides ruber, Globigerinoides sacculifer, Globigerinita glutinata, and Globorotalia menardii. The F2 assemblage has important contributions from Globigerinita glutinata, Globigerina bulloides, and Neogloboquadrina pachyderma (sinistral). The counting category with the highest scores in the F3 assemblage is Globorotalia menardii, followed by Neogloboquadrina dutertrei, Pulleniatina obliquiloculata, Globigerinoides sacculifer, and Globigerinoides ruber. The F4 assemblage is dominated by Globorotalia inflata, followed by lesser contributions from Neogloboquadrina pachyderma (dextral and sinistral). The F5 assemblage is quantitatively dominated by Neogloboquadrina pachyderma (sinistral), Globigerina bulloides, and Globigerinita glutinata. Based on the geographic distribution of characteristic species, we categorize the five primary factors as subtropical (F1), boundary current (F2), tropical (F3), transitional between subtropical and subpolar (F4), and polar-subpolar (F5).

Calibration of the five modern factors to February and August SST resulted in two 20-term equations and an intercept that related the five varimax assemblages to cold and warm season SST. Both seasons had an $\mathrm{R}^{2}$ value of 0.98 and standard errors of $1.2^{\circ} \mathrm{C}$ (February) and $1.4^{\circ} \mathrm{C}$ (August). Collectively, these equations are referred to as GSF36. To apply GSF36 to the mid-Piacenzian planktic foraminifer assemblages, the raw faunal data were grouped into 33 counting categories like the core-top data. Some Pliocene taxa, however, are now extinct and were not explicitly included in the counting categories. We made the assumption that closely related taxa (ancestor-descendent pairs) have similar ecological tolerances and preferences (see Dowsett and Poore, 1990; Dowsett, 1991; Kucera and Schönfeld, 2007; Waterson and others, 2017). 
Table 3. Varimax assemblage description matrix showing the relative importance of each of the 33 variables (planktic foraminifer species) to each of the five primary factors (F1-F5). Together, these five factors explain 92 percent of the planktic foraminifer assemblage variance in the modern core-top data.

[The strongest associations between variables and primary factors are shown in bold.]

\begin{tabular}{|c|c|c|c|c|c|}
\hline \multirow{2}{*}{ Variable (planktic foraminifer species) } & \multicolumn{5}{|c|}{ Primary factor } \\
\hline & F1 & F2 & F3 & F4 & F5 \\
\hline Globigerinoides ruber & 5.1572 & -0.3653 & -0.4205 & 0.5170 & -0.0123 \\
\hline Globigerinoides sacculifer & 1.9042 & -0.6802 & 1.0439 & -0.2810 & 0.5983 \\
\hline Globigerinita glutinata & 1.0232 & 4.3010 & -0.2453 & -0.3559 & -1.6200 \\
\hline Globigerinella aequilateralis & 0.5664 & -0.2044 & 0.4744 & 0.0879 & 0.1171 \\
\hline Neogloboquadrina dutertrei & 0.6545 & 0.2719 & 2.5131 & -0.1245 & 0.2019 \\
\hline Globigerina calida & 0.3925 & -0.0649 & 0.0725 & 0.0994 & 0.0162 \\
\hline Globigerina bulloides & -0.2865 & 3.5113 & 0.0108 & 0.8992 & 3.2025 \\
\hline Pulleniatina obliquiloculata & 0.3784 & 0.1180 & 1.0520 & -0.2044 & 0.0629 \\
\hline Globigerinoides conglobatus & 0.3098 & -0.2324 & 0.3020 & -0.0118 & 0.1368 \\
\hline $\begin{array}{l}\text { Globorotalia truncatulinoides } \\
\text { (dextral) }\end{array}$ & 0.1357 & -0.1214 & -0.0292 & 0.2808 & -0.0518 \\
\hline Globigerina falconensis & 0.0263 & 0.5766 & -0.1313 & 0.4136 & -0.3587 \\
\hline Globorotaloides hexagona & 0.2442 & 0.0401 & 0.1146 & -0.0968 & 0.0635 \\
\hline Globigerinoides tennellus & 0.1884 & 0.1444 & -0.0766 & -0.0329 & -0.0443 \\
\hline Globigerina rubescens & 0.1397 & 0.1255 & -0.0532 & -0.0199 & -0.0407 \\
\hline Orbulina universa & 0.1127 & -0.0255 & 0.0174 & 0.1606 & -0.0112 \\
\hline $\begin{array}{l}\text { Globorotalia truncatulinoides } \\
\quad \text { (sinistral) }\end{array}$ & 0.0006 & -0.0345 & -0.0395 & 0.8594 & 0.1265 \\
\hline Neogloboquadrina conglomerata & 0.1853 & -0.2252 & 0.6082 & -0.0789 & 0.2340 \\
\hline Globorotalia scitula & 0.0780 & 0.0106 & -0.0135 & 0.0783 & -0.0115 \\
\hline Beella digitata & 0.0444 & -0.0490 & 0.2092 & 0.0704 & -0.0131 \\
\hline $\begin{array}{l}\text { Neogloboquadrina pachyderma } \\
\text { (sinistral) }\end{array}$ & 0.2318 & -0.8255 & 0.0238 & -0.5625 & 4.3590 \\
\hline Globorotalia crassaformis & 0.0298 & -0.0242 & 0.0431 & 0.0088 & 0.0104 \\
\hline Candeina nitida & 0.0215 & -0.0104 & -0.0037 & 0.0029 & 0.0074 \\
\hline Globorotalia menardii flexuosa & 0.0091 & 0.0013 & 0.0315 & -0.0041 & 0.0059 \\
\hline Globorotalia hirsuta & 0.0170 & -0.0381 & 0.0076 & 0.2196 & -0.0698 \\
\hline Sphaeroidinella dehiscens & 0.0029 & -0.0398 & 0.1684 & 0.0021 & 0.0237 \\
\hline Turborotalita humilis & 0.0046 & -0.0012 & 0.0002 & -0.0001 & 0.0012 \\
\hline Turborotalita quinqueloba & -0.0169 & 0.0181 & -0.0111 & 0.0255 & 0.3595 \\
\hline Hastigerina adamsi & 0.0021 & 0.0015 & -0.0010 & -0.0007 & 0.0002 \\
\hline Neogloboquadrina “du-pac" & -0.0847 & 0.0738 & 0.0456 & 0.6074 & 0.3578 \\
\hline Globorotalia tumida & -0.1253 & 0.0588 & 0.6445 & 0.0445 & -0.1128 \\
\hline Globorotalia menardii & -0.4219 & 0.2478 & 4.7976 & 0.1112 & -0.4091 \\
\hline $\begin{array}{l}\text { Neogloboquadrina pachyderma } \\
\text { (dextral) }\end{array}$ & -0.1449 & 0.0494 & 0.0284 & 1.2607 & -0.0976 \\
\hline Globorotalia inflata & -0.1979 & -0.4358 & 0.0786 & 5.3163 & -0.1430 \\
\hline Variance & 34.304 & 21.098 & 19.030 & 10.470 & 7.101 \\
\hline Cumulative variance & 34.304 & 55.402 & 74.432 & 84.902 & 92.003 \\
\hline
\end{tabular}




\section{Results and Discussion}

\section{Planktic Foraminiferal Assemblage Data}

Planktic foraminifer census data from the mid-Piacenzian Indian Ocean sites (Dowsett and others, 2015) can be accessed at the National Oceanic and Atmospheric Administration's National Centers for Environmental Information (NCEI) (Foley and others, 2015; https://www.ncdc.noaa.gov/paleo/study/19281). Figure 1 shows the geographic distribution of species in terms of mean percentage at each site, and figure 2 displays time series percent data for the mid-Piacenzian interval of each Indian Ocean site. In general, the mid-Piacenzian tropical Indian Ocean was characterized by an abundance of Globigerinoides species and Globorotalia menardii. Site 722, located in a tropical upwelling zone, was dominated by Globigerinita glutinata.
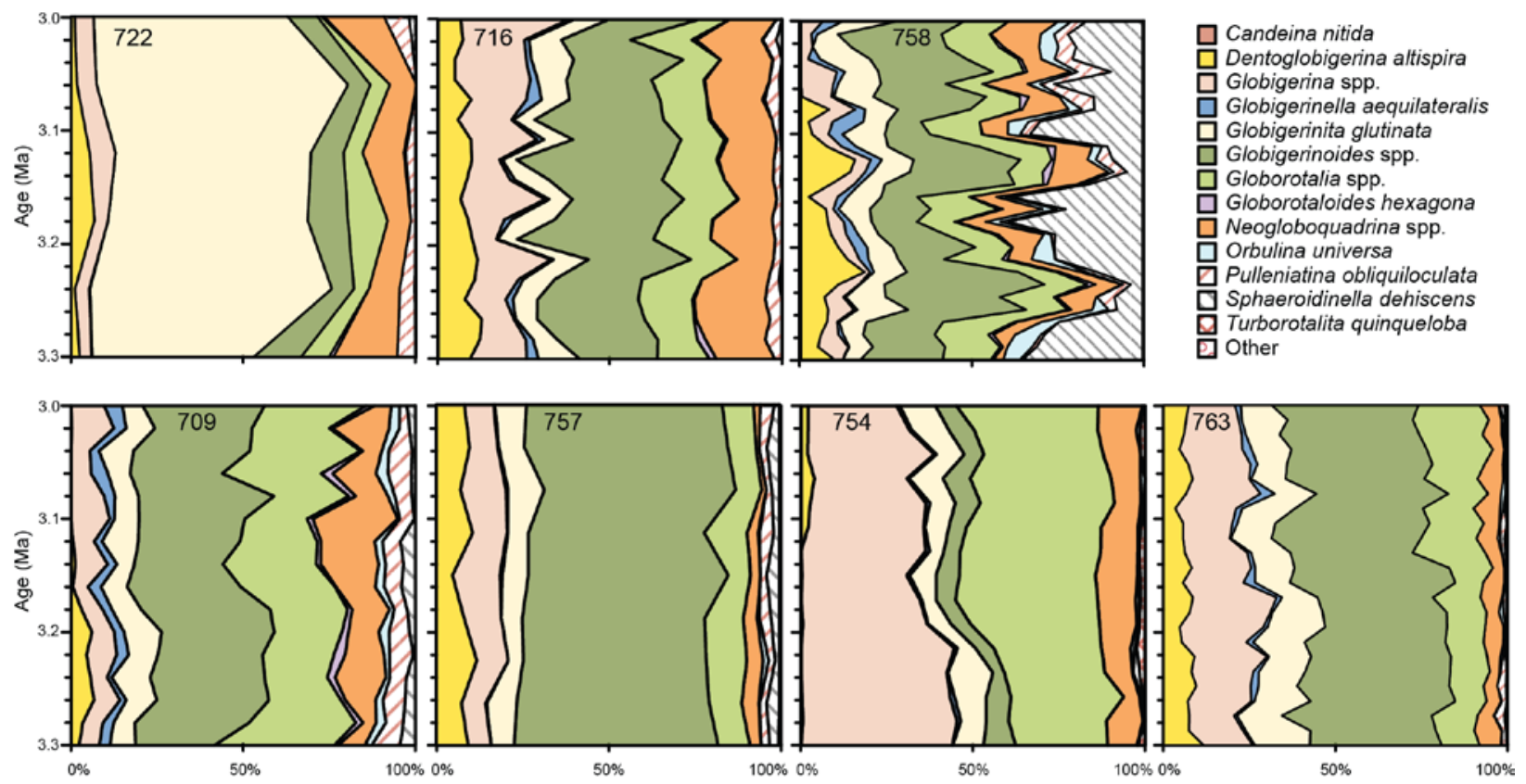

Figure 2. Time series plots of mid-Piacenzian planktic foraminifer species assemblages by percentage for Ocean Drilling Program sites 709, 716, 722, 754, 757, 758, and 763 in the Indian Ocean, arranged geographically.

\section{Modern Analog Technique}

The modern analog technique performed adequately at five of the seven low- to midlatitude Indian Ocean sites. Squared chord distances to the nearest modern analog are $\leq 0.34$ for all samples from sites 709, 716, 722, 757, and 763, with the vast majority within a distance of 0.20 (table 4). Cold and warm season SST estimates are shown in table 4. Most samples from ODP sites 754 and 758 showed higher dissimilarity, indicating an absence of analogous faunas in the modern Indian Ocean. Because faunal census data from sites 754 and 758 are predominantly non-analog assemblages, we have reduced confidence in the resulting SST estimates. 


\section{Factor Analytic Transfer Function}

Estimates of SST were obtained using GSF36 for mid-Piacenzian Indian Ocean samples from ODP sites 709, 716, 722, 754, 758, and 763 (fig. 3). Cold and warm season SST estimates are shown in table 4. GSF36 factors provide a quantitative picture of the assemblage composition of each sample. Factor loadings indicate a strong relationship between mid-Piacenzian samples from site 709 and both tropical and subtropical assemblages and a strong relationship between site 763 samples and subtropical assemblages. GSF36 functioned very well at these two sites with all samples returning a communality of $\geq 0.7$ (that is, 84 percent or more of the information was explained by the core-top factor model). For site 716, 74 percent of samples had acceptable communalities. All but one of these samples were tied to subtropical assemblages; the remaining sample (at $3.02 \mathrm{Ma}$ ) was more closely related to a tropical assemblage. Site 722 mid-Piacenzian samples are most closely related to boundary current assemblages, and site 754 samples are most closely related to transitional assemblages, although only 67 percent and 30 percent of samples, respectively, showed communalities $\geq 0.7$. GSF36 could deliver meaningful oceanographic estimates for only seven percent of site 758 samples; the remaining samples had no modern analogs. Those few samples, however, did show a clear relationship to subtropical assemblages.

Table 4. Cold and warm season sea surface temperature (SST) estimates for samples using the modern analog technique with squared chord distance (SCD) values and the factor analytic transfer function GSF36 with communalities.

[cm, centimeter; mbsf, meters below sea floor; Ma, million years ago; ${ }^{\circ} \mathrm{C}$, degree Celsius; ODP, Ocean Drilling Program]

\begin{tabular}{|c|c|c|c|c|c|c|c|c|}
\hline \multirow[b]{2}{*}{ Sample } & \multirow[b]{2}{*}{$\begin{array}{l}\text { Depth } \\
\text { (mbsf) }\end{array}$} & \multirow[b]{2}{*}{$\begin{array}{l}\text { Age } \\
\text { (Ma) }\end{array}$} & \multicolumn{3}{|c|}{ Modern analog technique } & \multicolumn{3}{|c|}{ Factor analytic transfer function } \\
\hline & & & $\begin{array}{c}\text { Cold SST } \\
\left({ }^{\circ} \mathrm{C}\right)\end{array}$ & $\begin{array}{c}\text { Warm SST } \\
\left({ }^{\circ} \mathrm{C}\right)\end{array}$ & SCD & $\begin{array}{c}\text { Cold SST } \\
\left({ }^{\circ} \mathrm{C}\right)\end{array}$ & $\begin{array}{c}\text { Warm SST } \\
\left({ }^{\circ} \mathrm{C}\right)\end{array}$ & $\begin{array}{c}\text { Commu } \\
\text { nality }\end{array}$ \\
\hline \multicolumn{9}{|c|}{ ODP site 709 , hole C } \\
\hline $4 \mathrm{H}-2,42-44 \mathrm{~cm}$ & 27.02 & 2.83 & 26.17 & 29.11 & 0.14 & 26.7 & 27.8 & 0.8886 \\
\hline $4 \mathrm{H}-2,57.5-59.5 \mathrm{~cm}$ & 27.18 & 2.85 & 26.17 & 29.11 & 0.11 & 26.7 & 27.4 & 0.9028 \\
\hline $4 \mathrm{H}-2,83-85 \mathrm{~cm}$ & 27.43 & 2.88 & 26.17 & 29.11 & 0.10 & 27.2 & 28.7 & 0.9095 \\
\hline $4 \mathrm{H}-2,102.5-104.5 \mathrm{~cm}$ & 27.63 & 2.90 & 26.17 & 29.11 & 0.10 & 27.3 & 28.3 & 0.8495 \\
\hline $4 \mathrm{H}-2,112.5-114.5 \mathrm{~cm}$ & 27.73 & 2.91 & 4.47 & 7.59 & 0.14 & 27.3 & 27.9 & 0.8852 \\
\hline $4 \mathrm{H}-2,132-134 \mathrm{~cm}$ & 27.92 & 2.93 & 26.17 & 29.11 & 0.10 & 27.7 & 28.2 & 0.8455 \\
\hline $4 \mathrm{H}-2,142-144 \mathrm{~cm}$ & 28.02 & 2.95 & 26.17 & 29.11 & 0.13 & 27.5 & 28.0 & 0.8988 \\
\hline $4 \mathrm{H}-3,2-4 \mathrm{~cm}$ & 28.12 & 2.96 & 26.17 & 29.11 & 0.06 & 27.7 & 28.6 & 0.9550 \\
\hline $4 \mathrm{H}-3,17-19 \mathrm{~cm}$ & 28.27 & 2.97 & 24.41 & 29.11 & 0.10 & 27.6 & 28.7 & 0.8547 \\
\hline $4 \mathrm{H}-3,33-35 \mathrm{~cm}$ & 28.43 & 2.99 & 26.17 & 29.11 & 0.15 & 27.1 & 27.7 & 0.9074 \\
\hline $4 \mathrm{H}-3,46-49 \mathrm{~cm}$ & 28.56 & 3.01 & 24.34 & 27.78 & 0.14 & 27.6 & 28.1 & 0.9262 \\
\hline $4 \mathrm{H}-3,57.5-59.5 \mathrm{~cm}$ & 28.68 & 3.02 & 26.17 & 29.11 & 0.12 & 27.3 & 27.9 & 0.9327 \\
\hline $4 \mathrm{H}-3,83-85 \mathrm{~cm}$ & 28.93 & 3.05 & 26.17 & 29.11 & 0.15 & 27.1 & 28.3 & 0.8523 \\
\hline $4 \mathrm{H}-3,93.5-95.5 \mathrm{~cm}$ & 29.04 & 3.06 & 24.77 & 25.00 & 0.12 & 28.5 & 29.2 & 0.8086 \\
\hline $4 \mathrm{H}-3,117.5-119.5 \mathrm{~cm}$ & 29.28 & 3.09 & 24.57 & 25.93 & 0.09 & 28.5 & 29.1 & 0.8345 \\
\hline $4 \mathrm{H}-3,132.5-134.5 \mathrm{~cm}$ & 29.43 & 3.11 & 25.47 & 27.06 & 0.11 & 27.6 & 28.6 & 0.8933 \\
\hline $4 \mathrm{H}-3,142-144 \mathrm{~cm}$ & 29.52 & 3.12 & 26.17 & 29.11 & 0.11 & 27.1 & 28.5 & 0.8226 \\
\hline $4 \mathrm{H}-4,7-9 \mathrm{~cm}$ & 29.67 & 3.14 & 26.17 & 29.11 & 0.14 & 27.7 & 28.5 & 0.7835 \\
\hline $4 \mathrm{H}-4,17-19 \mathrm{~cm}$ & 29.77 & 3.19 & 8.93 & 10.48 & 0.15 & 28.0 & 28.4 & 0.8178 \\
\hline $4 \mathrm{H}-4,32-34 \mathrm{~cm}$ & 29.92 & 3.25 & 26.17 & 29.11 & 0.15 & 27.9 & 28.7 & 0.7544 \\
\hline $4 \mathrm{H}-4,46.5-48.5 \mathrm{~cm}$ & 30.07 & 3.31 & 25.04 & 27.07 & 0.23 & 28.1 & 28.9 & 0.7894 \\
\hline
\end{tabular}


Table 4. Cold and warm season sea surface temperature (SST) estimates for samples using the modern analog technique with squared chord distance (SCD) values and the factor analytic transfer function GSF36 with communalities. - Continued

[cm, centimeter; mbsf, meters below sea floor; Ma, million years ago; ${ }^{\circ} \mathrm{C}$, degree Celsius; ODP, Ocean Drilling Program]

\begin{tabular}{|c|c|c|c|c|c|c|c|c|}
\hline \multirow[b]{2}{*}{ Sample } & \multirow[b]{2}{*}{$\begin{array}{l}\text { Depth } \\
\text { (mbsf) }\end{array}$} & \multirow[b]{2}{*}{$\begin{array}{l}\text { Age } \\
\text { (Ma) }\end{array}$} & \multicolumn{3}{|c|}{ Modern analog technique } & \multicolumn{3}{|c|}{ Factor analytic transfer function } \\
\hline & & & $\begin{array}{c}\text { Cold SST } \\
\left({ }^{\circ} \mathrm{C}\right)\end{array}$ & $\begin{array}{c}\text { Warm SST } \\
\left({ }^{\circ} \mathrm{C}\right)\end{array}$ & SCD & $\begin{array}{c}\text { Cold SST } \\
\left({ }^{\circ} \mathrm{C}\right)\end{array}$ & $\begin{array}{c}\text { Warm SST } \\
\left({ }^{\circ} \mathrm{C}\right)\end{array}$ & $\begin{array}{c}\text { Commu } \\
\text { nality }\end{array}$ \\
\hline \multicolumn{9}{|c|}{ ODP site 716 , hole B } \\
\hline $11-3,54-56 \mathrm{~cm}$ & 94.24 & 2.95 & 25.00 & 28.91 & 0.25 & 27.8 & 27.8 & 0.6248 \\
\hline $11-3,128-130 \mathrm{~cm}$ & 94.98 & 2.97 & 26.17 & 29.11 & 0.30 & 27.5 & 27.7 & 0.5587 \\
\hline $11-4,58-60 \mathrm{~cm}$ & 95.78 & 2.98 & 3.02 & 5.70 & 0.24 & 28.2 & 28.3 & 0.6876 \\
\hline $11-4,139-141 \mathrm{~cm}$ & 96.59 & 3.00 & 3.02 & 5.70 & 0.25 & 27.0 & 26.6 & 0.4764 \\
\hline $11-5,68-70 \mathrm{~cm}$ & 97.38 & 3.02 & 24.34 & 27.78 & 0.19 & 27.5 & 28.3 & 0.7561 \\
\hline $11-6,1-3 \mathrm{~cm}$ & 98.21 & 3.03 & 3.02 & 5.70 & 0.15 & 28.1 & 28.3 & 0.7555 \\
\hline $11-6,84-86 \mathrm{~cm}$ & 99.04 & 3.05 & 25.18 & 28.44 & 0.24 & 28.0 & 28.7 & 0.6858 \\
\hline $11-7,9-11 \mathrm{~cm}$ & 99.79 & 3.07 & 26.65 & 28.25 & 0.18 & 28.4 & 29.1 & 0.7260 \\
\hline $12-1,27-29 \mathrm{~cm}$ & 100.58 & 3.08 & 26.65 & 28.25 & 0.14 & 28.7 & 29.0 & 0.8381 \\
\hline $12-1,113-115 \mathrm{~cm}$ & 101.44 & 3.10 & 26.65 & 28.25 & 0.14 & 28.3 & 28.3 & 0.7944 \\
\hline $12-2,37-39 \mathrm{~cm}$ & 102.18 & 3.12 & 26.65 & 28.25 & 0.11 & 29.1 & 29.3 & 0.8156 \\
\hline $12-2,117-119 \mathrm{~cm}$ & 102.98 & 3.13 & 26.65 & 28.25 & 0.15 & 28.0 & 28.8 & 0.8441 \\
\hline $12-3,53-55 \mathrm{~cm}$ & 103.84 & 3.15 & 3.02 & 5.70 & 0.16 & 28.4 & 28.8 & 0.7780 \\
\hline $12-3,127-129 \mathrm{~cm}$ & 104.58 & 3.17 & 26.41 & 28.68 & 0.22 & 27.4 & 27.6 & 0.7948 \\
\hline $12-4,57.5-59.5 \mathrm{~cm}$ & 105.39 & 3.18 & 26.65 & 28.25 & 0.18 & 28.4 & 28.1 & 0.8648 \\
\hline $12-4,137.5-139.5 \mathrm{~cm}$ & 106.19 & 3.20 & 3.02 & 5.70 & 0.20 & 28.2 & 28.4 & 0.7339 \\
\hline $12-5,67-69 \mathrm{~cm}$ & 106.98 & 3.22 & 26.65 & 28.25 & 0.20 & 27.8 & 28.5 & 0.8343 \\
\hline $12-6,3-5 \mathrm{~cm}$ & 107.84 & 3.23 & 26.41 & 28.68 & 0.27 & 27.7 & 27.2 & 0.6949 \\
\hline $12-6,84-86 \mathrm{~cm}$ & 108.65 & 3.25 & 26.65 & 28.25 & 0.22 & 28.2 & 27.9 & 0.7693 \\
\hline $12-7,7-9 \mathrm{~cm}$ & 109.38 & 3.27 & 25.83 & 28.58 & 0.18 & 28.6 & 29.0 & 0.8309 \\
\hline $13-1,97.5-99.5 \mathrm{~cm}$ & 110.99 & 3.30 & 3.02 & 5.70 & 0.18 & 27.3 & 27.9 & 0.8107 \\
\hline $13-2,34-36 \mathrm{~cm}$ & 111.85 & 3.32 & 25.50 & 28.02 & 0.24 & 27.8 & 27.1 & 0.7653 \\
\hline $13-2,113-115 \mathrm{~cm}$ & 112.64 & 3.33 & 24.34 & 27.78 & 0.21 & 27.9 & 28.4 & 0.7810 \\
\hline \multicolumn{9}{|c|}{ ODP site 722 , hole $A$} \\
\hline $10 \mathrm{X}-3,10-12 \mathrm{~cm}$ & 88.61 & 2.85 & 6.06 & 7.63 & 0.18 & 27.3 & 27.4 & 0.7306 \\
\hline $10 \mathrm{X}-3,110-112 \mathrm{~cm}$ & 89.61 & 2.88 & 24.86 & 26.40 & 0.13 & 27.7 & 28.2 & 0.5446 \\
\hline $10 \mathrm{X}-4,10-12 \mathrm{~cm}$ & 90.11 & 2.90 & 26.58 & 26.87 & 0.17 & 28.0 & 28.2 & 0.6819 \\
\hline $10 \mathrm{X}-4,110-112 \mathrm{~cm}$ & 91.11 & 2.94 & 27.47 & 27.60 & 0.27 & 26.7 & 27.5 & 0.6650 \\
\hline $10 X-5,10-12 \mathrm{~cm}$ & 91.61 & 2.96 & 6.06 & 7.63 & 0.34 & 26.2 & 28.1 & 0.5525 \\
\hline $11 \mathrm{X}-1,12-14 \mathrm{~cm}$ & 95.33 & 3.09 & 24.44 & 24.78 & 0.22 & 25.6 & 28.4 & 0.6898 \\
\hline $11 \mathrm{X}-1,108-110 \mathrm{~cm}$ & 96.29 & 3.11 & 24.44 & 24.78 & 0.17 & 23.3 & 29.1 & 0.7410 \\
\hline $11 \mathrm{X}-2,8-10 \mathrm{~cm}$ & 96.79 & 3.13 & 25.04 & 25.93 & 0.18 & 24.0 & 29.0 & 0.7231 \\
\hline $11 \mathrm{X}-2,112-114 \mathrm{~cm}$ & 97.83 & 3.16 & 6.06 & 7.63 & 0.28 & 23.9 & 28.6 & 0.7144 \\
\hline $11 \mathrm{X}-3,112-114 \mathrm{~cm}$ & 99.33 & 3.23 & 25.37 & 26.28 & 0.16 & 24.6 & 28.3 & 0.7322 \\
\hline \multicolumn{9}{|c|}{ ODP site 754 , hole A } \\
\hline $2-4,1-5 \mathrm{~cm}$ & 10.61 & 2.71 & 23.88 & 27.60 & 0.27 & 15.7 & 21.5 & 0.7461 \\
\hline $2-4,14-18 \mathrm{~cm}$ & 10.74 & 2.73 & 8.18 & 10.55 & 0.30 & 15.6 & 21.6 & 0.7435 \\
\hline $2-4,29-33 \mathrm{~cm}$ & 10.89 & 2.75 & 23.88 & 27.60 & 0.29 & 16.5 & 22.1 & 0.5860 \\
\hline $2-4,44-48 \mathrm{~cm}$ & 11.04 & 2.77 & 21.85 & 26.46 & 0.34 & 15.8 & 21.4 & 0.5626 \\
\hline $2-4,59-63 \mathrm{~cm}$ & 11.19 & 2.79 & 23.88 & 27.60 & 0.34 & 16.1 & 22.2 & 0.6246 \\
\hline
\end{tabular}


Table 4. Cold and warm season sea surface temperature (SST) estimates for samples using the modern analog technique with squared chord distance (SCD) values and the factor analytic transfer function GSF36 with communalities. - Continued

[cm, centimeter; mbsf, meters below sea floor; Ma, million years ago; ${ }^{\circ} \mathrm{C}$, degree Celsius; ODP, Ocean Drilling Program]

\begin{tabular}{|c|c|c|c|c|c|c|c|c|}
\hline \multirow[b]{2}{*}{ Sample } & \multirow[b]{2}{*}{$\begin{array}{l}\text { Depth } \\
\text { (mbsf) }\end{array}$} & \multirow[b]{2}{*}{$\begin{array}{l}\text { Age } \\
\text { (Ma) }\end{array}$} & \multicolumn{3}{|c|}{ Modern analog technique } & \multicolumn{3}{|c|}{ Factor analytic transfer function } \\
\hline & & & $\begin{array}{c}\text { Cold SST } \\
\left({ }^{\circ} \mathrm{C}\right)\end{array}$ & $\begin{array}{c}\text { Warm SST } \\
\left({ }^{\circ} \mathrm{C}\right)\end{array}$ & SCD & $\begin{array}{c}\text { Cold SST } \\
\left({ }^{\circ} \mathrm{C}\right)\end{array}$ & $\begin{array}{c}\text { Warm SST } \\
\left({ }^{\circ} \mathrm{C}\right)\end{array}$ & $\begin{array}{l}\text { Commu } \\
\text { nality }\end{array}$ \\
\hline \multicolumn{9}{|c|}{ ODP site 754 , hole A-Continued } \\
\hline $2-4,74-78 \mathrm{~cm}$ & 11.34 & 2.82 & 23.88 & 27.60 & 0.24 & 16.8 & 22.7 & 0.8451 \\
\hline 2-4, 89-93 cm & 11.49 & 2.84 & 23.88 & 27.60 & 0.26 & 16.6 & 23.4 & 0.7993 \\
\hline $2-4,104-108 \mathrm{~cm}$ & 11.64 & 2.86 & 23.88 & 27.60 & 0.39 & 16.5 & 22.7 & 0.5605 \\
\hline $2-4,119-123 \mathrm{~cm}$ & 11.79 & 2.89 & 23.88 & 27.60 & 0.39 & 16.0 & 22.1 & 0.6015 \\
\hline $2-4,134-138 \mathrm{~cm}$ & 11.94 & 2.91 & 23.88 & 27.60 & 0.33 & 16.4 & 22.9 & 0.7101 \\
\hline $2-5,0-4 \mathrm{~cm}$ & 12.10 & 2.93 & 23.88 & 27.60 & 0.38 & 15.7 & 21.5 & 0.6303 \\
\hline $2-5,14-18 \mathrm{~cm}$ & 12.24 & 2.95 & 23.88 & 27.60 & 0.39 & 17.1 & 21.7 & 0.5332 \\
\hline $2-5,29-33 \mathrm{~cm}$ & 12.39 & 2.97 & 23.88 & 27.60 & 0.32 & 16.7 & 22.3 & 0.5966 \\
\hline $2-5,44-48 \mathrm{~cm}$ & 12.54 & 3.00 & 23.88 & 27.60 & 0.31 & 16.7 & 22.4 & 0.7006 \\
\hline $2-5,59-63 \mathrm{~cm}$ & 12.69 & 3.02 & 23.88 & 27.60 & 0.36 & 17.1 & 22.4 & 0.5763 \\
\hline $2-5,74-78 \mathrm{~cm}$ & 12.84 & 3.04 & 23.88 & 27.60 & 0.39 & 17.3 & 22.4 & 0.4549 \\
\hline 2-5, 89-93 cm & 12.99 & 3.06 & 23.88 & 27.60 & 0.33 & 16.4 & 22.6 & 0.6914 \\
\hline $2-5,104-108 \mathrm{~cm}$ & 13.14 & 3.09 & 23.88 & 27.60 & 0.36 & 16.3 & 22.1 & 0.6423 \\
\hline $2-5,119-123 \mathrm{~cm}$ & 13.29 & 3.11 & 23.88 & 27.60 & 0.40 & 16.4 & 22.2 & 0.5992 \\
\hline $2-5,134-138 \mathrm{~cm}$ & 13.44 & 3.13 & 23.88 & 27.60 & 0.39 & 16.2 & 21.5 & 0.6807 \\
\hline $2-6,0-4 \mathrm{~cm}$ & 13.60 & 3.15 & 23.88 & 27.60 & 0.36 & 16.8 & 22.4 & 0.6412 \\
\hline $2-6,14-18 \mathrm{~cm}$ & 13.74 & 3.17 & 23.88 & 27.60 & 0.37 & 16.1 & 21.9 & 0.7319 \\
\hline $2-6,29-33 \mathrm{~cm}$ & 13.89 & 3.20 & 23.88 & 27.60 & 0.36 & 16.2 & 21.6 & 0.7102 \\
\hline $2-6,44-48 \mathrm{~cm}$ & 14.04 & 3.22 & 21.85 & 26.46 & 0.47 & 15.2 & 20.9 & 0.4945 \\
\hline $2-6,59-63 \mathrm{~cm}$ & 14.19 & 3.24 & 23.88 & 27.60 & 0.53 & 15.6 & 20.5 & 0.4392 \\
\hline $2-6,74-78 \mathrm{~cm}$ & 14.34 & 3.26 & 23.88 & 27.60 & 0.39 & 15.9 & 21.3 & 0.4890 \\
\hline 2-6, 89-93 cm & 14.49 & 3.29 & 23.88 & 27.60 & 0.37 & 16.1 & 21.5 & 0.4400 \\
\hline $2-6,104-108 \mathrm{~cm}$ & 14.64 & 3.31 & 23.88 & 27.60 & 0.41 & 16.0 & 21.3 & 0.4663 \\
\hline $2-6,119-123 \mathrm{~cm}$ & 14.79 & 3.33 & 23.88 & 27.60 & 0.41 & 15.8 & 21.5 & 0.5134 \\
\hline $2-6,134-138 \mathrm{~cm}$ & 14.94 & 3.35 & 23.88 & 27.60 & 0.42 & 16.4 & 22.1 & 0.4515 \\
\hline \multicolumn{9}{|c|}{ ODP site 757 , hole B } \\
\hline $3 \mathrm{H}-4,24-26 \mathrm{~cm}$ & 18.75 & 2.91 & 23.32 & 25.96 & 0.11 & 25.2 & 27.5 & 0.8788 \\
\hline $3 \mathrm{H}-4,54-56 \mathrm{~cm}$ & 19.05 & 2.96 & 25.99 & 27.12 & 0.12 & 25.0 & 27.2 & 0.8568 \\
\hline $3 \mathrm{H}-4,84-86 \mathrm{~cm}$ & 19.35 & 3.00 & 0.76 & 3.01 & 0.11 & 24.8 & 27.6 & 0.8879 \\
\hline $3 \mathrm{H}-4,114-116 \mathrm{~cm}$ & 19.65 & 3.03 & 25.99 & 27.12 & 0.10 & 24.0 & 27.3 & 0.9330 \\
\hline $3 \mathrm{H}-4,144-146 \mathrm{~cm}$ & 19.95 & 3.07 & 0.76 & 3.01 & 0.12 & 24.3 & 27.5 & 0.9034 \\
\hline $3 \mathrm{H}-5,24-26 \mathrm{~cm}$ & 20.25 & 3.11 & 25.33 & 25.99 & 0.09 & 25.1 & 27.7 & 0.8694 \\
\hline $3 \mathrm{H}-5,57-59 \mathrm{~cm}$ & 20.58 & 3.15 & 0.76 & 3.01 & 0.11 & 24.3 & 27.5 & 0.9100 \\
\hline $3 \mathrm{H}-5,84-86 \mathrm{~cm}$ & 20.85 & 3.19 & 26.65 & 28.25 & 0.12 & 25.3 & 27.9 & 0.7976 \\
\hline $3 \mathrm{H}-5,109-111 \mathrm{~cm}$ & 21.10 & 3.22 & 25.33 & 25.99 & 0.17 & 24.7 & 27.6 & 0.7526 \\
\hline $3 \mathrm{H}-6,11.5-13.5 \mathrm{~cm}$ & 21.63 & 3.28 & 25.99 & 27.12 & 0.11 & 25.2 & 27.9 & 0.8023 \\
\hline $3 \mathrm{H}-6,29-31 \mathrm{~cm}$ & 21.80 & 3.31 & 25.99 & 27.12 & 0.12 & 25.1 & 27.9 & 0.7453 \\
\hline 3H-6, 63-65 cm & 22.14 & 3.35 & 25.33 & 25.99 & 0.15 & 24.7 & 27.7 & 0.6974 \\
\hline
\end{tabular}


Table 4. Cold and warm season sea surface temperature (SST) estimates for samples using the modern analog technique with squared chord distance (SCD) values and the factor analytic transfer function GSF36 with communalities. - Continued

[cm, centimeter; mbsf, meters below sea floor; Ma, million years ago; ${ }^{\circ} \mathrm{C}$, degree Celsius; ODP, Ocean Drilling Program]

\begin{tabular}{|c|c|c|c|c|c|c|c|c|}
\hline \multirow[b]{2}{*}{ Sample } & \multirow[b]{2}{*}{$\begin{array}{l}\text { Depth } \\
\text { (mbsf) }\end{array}$} & \multirow[b]{2}{*}{$\begin{array}{l}\text { Age } \\
\text { (Ma) }\end{array}$} & \multicolumn{3}{|c|}{ Modern analog technique } & \multicolumn{3}{|c|}{ Factor analytic transfer function } \\
\hline & & & $\begin{array}{c}\text { Cold SST } \\
\left({ }^{\circ} \mathrm{C}\right)\end{array}$ & $\begin{array}{c}\text { Warm SST } \\
\left({ }^{\circ} \mathrm{C}\right)\end{array}$ & SCD & $\begin{array}{c}\text { Cold SST } \\
\left({ }^{\circ} \mathrm{C}\right)\end{array}$ & $\begin{array}{c}\text { Warm SST } \\
\left({ }^{\circ} \mathrm{C}\right)\end{array}$ & $\begin{array}{c}\text { Commu } \\
\text { nality }\end{array}$ \\
\hline \multicolumn{9}{|c|}{ ODP site 758, hole $A$} \\
\hline $5 \mathrm{H}-3,7-9 \mathrm{~cm}$ & 37.88 & 2.92 & 24.63 & 28.16 & 0.40 & 27.0 & 27.0 & 0.4274 \\
\hline $5 \mathrm{H}-3,24-25 \mathrm{~cm}$ & 38.04 & 2.93 & 21.41 & 25.76 & 0.46 & 25.1 & 24.9 & 0.1294 \\
\hline $5 \mathrm{H}-3,44-46 \mathrm{~cm}$ & 38.24 & 2.95 & 4.47 & 7.59 & 0.31 & 28.6 & 29.0 & 0.5675 \\
\hline $5 \mathrm{H}-3,65-67 \mathrm{~cm}$ & 38.45 & 2.96 & 4.47 & 7.59 & 0.31 & 29.0 & 28.0 & 0.4825 \\
\hline 5H-3, 79-81 cm & 38.59 & 2.97 & 8.93 & 10.48 & 0.35 & 28.9 & 28.7 & 0.4640 \\
\hline $5 \mathrm{H}-3,98.5-100.5 \mathrm{~cm}$ & 38.79 & 2.99 & 26.17 & 29.11 & 0.30 & 28.6 & 28.9 & 0.4424 \\
\hline $5 \mathrm{H}-3,126.5-128.5 \mathrm{~cm}$ & 39.07 & 3.00 & 24.63 & 28.16 & 0.18 & 30.1 & 29.5 & 0.7454 \\
\hline $5 \mathrm{H}-3,142.5-144.5 \mathrm{~cm}$ & 39.23 & 3.02 & 7.87 & 9.32 & 0.37 & 27.7 & 28.3 & 0.2704 \\
\hline $5 \mathrm{H}-4,9.5-11.5 \mathrm{~cm}$ & 39.40 & 3.03 & 24.77 & 25.00 & 0.22 & 29.9 & 29.6 & 0.6747 \\
\hline $5 \mathrm{H}-4,24.5-25.5 \mathrm{~cm}$ & 39.55 & 3.05 & 4.47 & 7.59 & 0.30 & 28.9 & 28.2 & 0.4345 \\
\hline $5 \mathrm{H}-4,48-52 \mathrm{~cm}$ & 39.78 & 3.06 & 25.61 & 27.20 & 0.50 & 25.3 & 25.6 & 0.1214 \\
\hline $5 \mathrm{H}-4,67-69 \mathrm{~cm}$ & 39.97 & 3.07 & 21.41 & 25.76 & 0.46 & 26.3 & 27.3 & 0.1793 \\
\hline $5 \mathrm{H}-4,83-85 \mathrm{~cm}$ & 40.13 & 3.08 & 27.67 & 28.44 & 0.24 & 30.2 & 30.5 & 0.5562 \\
\hline $5 \mathrm{H}-4,107-109 \mathrm{~cm}$ & 40.37 & 3.10 & 24.74 & 28.17 & 0.28 & 29.2 & 28.8 & 0.4170 \\
\hline $5 \mathrm{H}-4,127.5-129.5 \mathrm{~cm}$ & 40.58 & 3.11 & 24.63 & 28.16 & 0.18 & 30.5 & 30.2 & 0.6852 \\
\hline $5 \mathrm{H}-5,5.5-7.5 \mathrm{~cm}$ & 40.86 & 3.14 & 26.65 & 28.25 & 0.20 & 29.8 & 28.3 & 0.6942 \\
\hline $5 \mathrm{H}-5,22-24 \mathrm{~cm}$ & 41.02 & 3.15 & 21.41 & 25.76 & 0.53 & 24.5 & 25.4 & 0.1011 \\
\hline $5 \mathrm{H}-5,37-39 \mathrm{~cm}$ & 41.17 & 3.16 & 6.70 & 9.04 & 0.39 & 28.6 & 28.4 & 0.3547 \\
\hline $5 \mathrm{H}-5,67-69 \mathrm{~cm}$ & 41.47 & 3.18 & 25.42 & 27.39 & 0.55 & 23.7 & 23.9 & 0.0824 \\
\hline $5 \mathrm{H}-5,82-84 \mathrm{~cm}$ & 41.62 & 3.20 & 24.86 & 27.13 & 0.39 & 28.2 & 27.9 & 0.2662 \\
\hline 5H-5, 95-97 cm & 41.75 & 3.21 & 21.41 & 25.76 & 0.41 & 27.3 & 27.0 & 0.2374 \\
\hline $5 \mathrm{H}-5,123-125 \mathrm{~cm}$ & 42.03 & 3.23 & 7.87 & 9.32 & 0.46 & 26.1 & 26.3 & 0.1610 \\
\hline $5 \mathrm{H}-5,142.5-144.5 \mathrm{~cm}$ & 42.23 & 3.24 & 28.30 & 28.62 & 0.30 & 28.9 & 28.7 & 0.3677 \\
\hline $5 \mathrm{H}-6,5.5-7.5 \mathrm{~cm}$ & 42.36 & 3.27 & 26.65 & 28.25 & 0.24 & 29.8 & 29.0 & 0.5377 \\
\hline $5 \mathrm{H}-6,27-29 \mathrm{~cm}$ & 42.57 & 3.29 & 26.65 & 28.25 & 0.15 & 30.2 & 30.0 & 0.7530 \\
\hline $5 \mathrm{H}-6,47.5-48.5 \mathrm{~cm}$ & 42.78 & 3.31 & 25.33 & 25.99 & 0.21 & 29.9 & 29.9 & 0.5781 \\
\hline $5 \mathrm{H}-6,65.5-67.5 \mathrm{~cm}$ & 42.96 & 3.33 & 8.93 & 10.48 & 0.47 & 27.8 & 27.9 & 0.2418 \\
\hline 5H-6, 93-95 cm & 43.23 & 3.35 & 21.41 & 25.76 & 0.42 & 27.8 & 27.5 & 0.2484 \\
\hline $5 \mathrm{H}-6,107.5-109.5 \mathrm{~cm}$ & 43.38 & 3.36 & 27.01 & 28.24 & 0.50 & 27.5 & 27.8 & 0.2105 \\
\hline $5 \mathrm{H}-6,122.5-124.5 \mathrm{~cm}$ & 43.53 & 3.38 & 21.36 & 25.84 & 0.53 & 26.7 & 26.5 & 0.2035 \\
\hline \multicolumn{9}{|c|}{ ODP site 763 , hole $A$} \\
\hline 7-7, 9-11 cm & 61.50 & 2.94 & 3.02 & 5.70 & 0.14 & 24.4 & 26.5 & 0.8475 \\
\hline $7-7,45-47 \mathrm{~cm}$ & 61.86 & 2.96 & 0.76 & 3.01 & 0.10 & 24.7 & 27.1 & 0.8974 \\
\hline 8-1, 9-11 cm & 62.00 & 2.98 & 26.65 & 28.25 & 0.13 & 26.3 & 28.0 & 0.8261 \\
\hline $8-1,26.5-28.5 \mathrm{~cm}$ & 62.18 & 2.99 & 3.02 & 5.70 & 0.14 & 26.2 & 27.6 & 0.8057 \\
\hline $8-1,43.5-45.5 \mathrm{~cm}$ & 62.35 & 3.00 & 1.89 & 4.36 & 0.10 & 26.1 & 27.7 & 0.8740 \\
\hline $8-1,66-68 \mathrm{~cm}$ & 62.57 & 3.01 & 26.41 & 28.68 & 0.11 & 26.6 & 27.8 & 0.8673 \\
\hline 8-1, 87-89 cm & 62.78 & 3.01 & 3.02 & 5.70 & 0.15 & 25.6 & 26.6 & 0.8110 \\
\hline 8-1, 106-108 cm & 62.97 & 3.02 & 24.71 & 27.76 & 0.13 & 26.4 & 27.6 & 0.8576 \\
\hline 8-1, 129-131 cm & 63.20 & 3.03 & 3.02 & 5.70 & 0.10 & 26.0 & 27.3 & 0.8256 \\
\hline $8-1,146.5-148.5 \mathrm{~cm}$ & 63.38 & 3.04 & 3.02 & 5.70 & 0.11 & 27.1 & 28.3 & 0.8775 \\
\hline 8-2, 9-11 cm & 63.50 & 3.05 & 25.71 & 26.63 & 0.13 & 26.4 & 27.7 & 0.8624 \\
\hline
\end{tabular}


Table 4. Cold and warm season sea surface temperature (SST) estimates for samples using the modern analog technique with squared chord distance (SCD) values and the factor analytic transfer function GSF36 with communalities. - Continued

[cm, centimeter; mbsf, meters below sea floor; Ma, million years ago; ${ }^{\circ} \mathrm{C}$, degree Celsius; ODP, Ocean Drilling Program]

\begin{tabular}{|c|c|c|c|c|c|c|c|c|}
\hline \multirow[b]{2}{*}{ Sample } & \multirow[b]{2}{*}{$\begin{array}{l}\text { Depth } \\
\text { (mbsf) }\end{array}$} & \multirow[b]{2}{*}{$\begin{array}{l}\text { Age } \\
(\mathrm{Ma})\end{array}$} & \multicolumn{3}{|c|}{ Modern analog technique } & \multicolumn{3}{|c|}{ Factor analytic transfer function } \\
\hline & & & $\begin{array}{c}\text { Cold SST } \\
\left({ }^{\circ} \mathrm{C}\right)\end{array}$ & $\begin{array}{c}\text { Warm SST } \\
\left({ }^{\circ} \mathrm{C}\right)\end{array}$ & SCD & $\begin{array}{c}\text { Cold SST } \\
\left({ }^{\circ} \mathrm{C}\right)\end{array}$ & $\begin{array}{c}\text { Warm SST } \\
\left({ }^{\circ} \mathrm{C}\right)\end{array}$ & $\begin{array}{c}\text { Commu } \\
\text { nality }\end{array}$ \\
\hline \multicolumn{9}{|c|}{ ODP site 763 , hole $\mathrm{A}-$ Continued } \\
\hline $8-2,27-29 \mathrm{~cm}$ & 63.68 & 3.06 & 3.02 & 5.70 & 0.09 & 26.5 & 27.9 & 0.8217 \\
\hline $8-2,45-47 \mathrm{~cm}$ & 63.86 & 3.07 & 3.02 & 5.70 & 0.13 & 26.8 & 27.7 & 0.8733 \\
\hline $8-2,66-68 \mathrm{~cm}$ & 64.07 & 3.08 & 25.71 & 26.63 & 0.11 & 26.0 & 27.7 & 0.8771 \\
\hline $8-2,87-89 \mathrm{~cm}$ & 64.28 & 3.08 & 26.65 & 28.25 & 0.10 & 25.8 & 27.4 & 0.8874 \\
\hline 8-2, 105-107 cm & 64.46 & 3.09 & 3.02 & 5.70 & 0.13 & 26.0 & 27.1 & 0.8332 \\
\hline $8-2,124-126 \mathrm{~cm}$ & 64.65 & 3.10 & 26.65 & 28.25 & 0.09 & 26.4 & 28.0 & 0.8938 \\
\hline $8-2,145-147 \mathrm{~cm}$ & 64.86 & 3.11 & 3.02 & 5.70 & 0.06 & 26.3 & 28.2 & 0.9282 \\
\hline $8-3,19-21 \mathrm{~cm}$ & 65.10 & 3.12 & 3.02 & 5.70 & 0.11 & 26.8 & 27.8 & 0.7976 \\
\hline $8-3,43-45 \mathrm{~cm}$ & 65.34 & 3.12 & 3.02 & 5.70 & 0.13 & 25.8 & 27.2 & 0.7903 \\
\hline $8-3,59-61 \mathrm{~cm}$ & 65.50 & 3.13 & 3.02 & 5.70 & 0.08 & 26.7 & 27.5 & 0.8616 \\
\hline $8-3,77.5-79.5 \mathrm{~cm}$ & 65.69 & 3.14 & 3.02 & 5.70 & 0.11 & 26.2 & 27.5 & 0.8776 \\
\hline $8-3,100-102 \mathrm{~cm}$ & 65.91 & 3.15 & 3.02 & 5.70 & 0.11 & 25.4 & 27.4 & 0.8534 \\
\hline $8-3,119-121 \mathrm{~cm}$ & 66.10 & 3.16 & 3.02 & 5.70 & 0.09 & 26.9 & 28.1 & 0.8784 \\
\hline 8-3, 139-141 cm & 66.30 & 3.17 & 26.65 & 28.25 & 0.09 & 25.9 & 27.9 & 0.9021 \\
\hline $8-4,9-11 \mathrm{~cm}$ & 66.50 & 3.18 & 3.02 & 5.70 & 0.07 & 26.2 & 27.7 & 0.9190 \\
\hline $8-4,27-29 \mathrm{~cm}$ & 66.68 & 3.19 & 26.65 & 28.25 & 0.07 & 26.1 & 27.7 & 0.9253 \\
\hline $8-4,47-49 \mathrm{~cm}$ & 66.88 & 3.20 & 3.02 & 5.70 & 0.09 & 26.0 & 27.2 & 0.9222 \\
\hline $8-4,79-81 \mathrm{~cm}$ & 67.20 & 3.22 & 3.02 & 5.70 & 0.08 & 25.0 & 27.2 & 0.9402 \\
\hline
\end{tabular}

Site 709

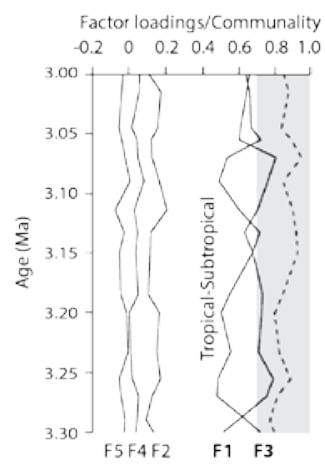

Site 716

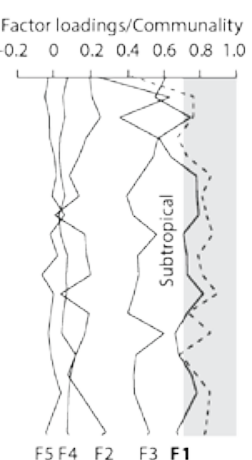

Site 722

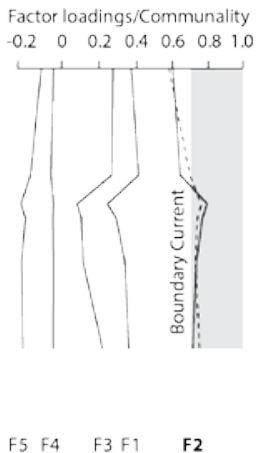

Site 754

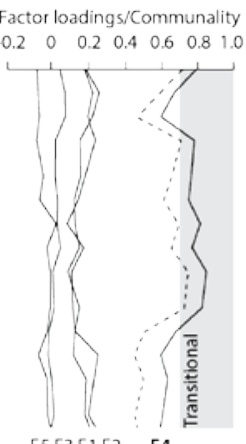

Site 758

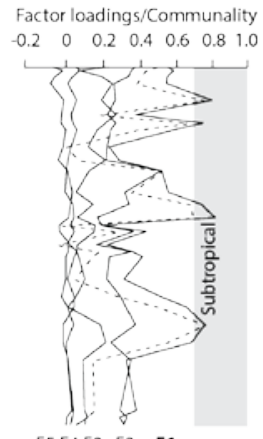

Site 763

Factor loadings/Communality $\begin{array}{ccccccc}-0.2 & 0 & 0.2 & 0.4 & 0.6 & 0.8 & 1.9\end{array}$

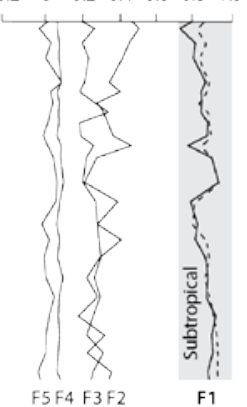

Figure 3. Downcore variation in factor loadings for F1 (subtropical), F2 (boundary current), F3 (tropical), F4 (transitional), and F5 (polar-subpolar) and downcore variation in communality (dashed line) for Ocean Drilling Program sites $709,716,722,754,758$, and 763 . Gray band represents the region of acceptable communality of $\geq 0.7$. 


\section{Summary and Conclusions}

The modern analog technique performed better than the factor analytic transfer function GSF36 in estimating SST within acceptable confidence limits (that is, SCD values $<0.2$ or communality values $\geq 0.7$ ) from these Indian Ocean samples. Despite success in estimating sea surface temperature for most mid-Piacenzian Indian Ocean samples, some assemblages from sites 716, 722, 754, and 758 were characterized as having no modern analogs and could not be characterized by GSF36 in terms of assemblages found in the modern ocean. In addition to the lack of good analogs, many samples throughout the Indian Ocean dataset were linked to modern analogs from various regions with diverse environmental characteristics. For example, a single site 763 sample at 3.05 Ma returned modern analogs of 0.13 SCD from both the tropical central Indian Ocean $\left(27.5^{\circ} \mathrm{C}\right)$ and the high-latitude south Atlantic Ocean $\left(4.4^{\circ} \mathrm{C}\right)$. Both modern samples are technically good analogs, but it is clear that temperature is not the common factor.

Future studies will focus on these non-analog assemblages, considering the degree of dissolution, the possibility of a non-analog Piacenzian environment, and the assumption of stationarity - the notion that ecological tolerances do not change with time. The assumption of stationarity appears to be reasonable: both techniques, for example, have been used to estimate SST in the mid-Piacenzian Atlantic and Pacific Oceans with much success (for example, Dowsett and others, 2009). While temperature is the single strongest factor in determining the relative composition of modern and most fossil foraminiferal assemblages, other factors such as surface productivity have been shown to have a strong effect (for example, Robinson and others, 2008) and may be influencing these Indian Ocean assemblages. Finally, a mixed layer environment of the past that does not exist today, with warmer surface temperatures or enhanced seasonal salinity stratification, for example, could explain the non-analog assemblages.

\section{Acknowledgments}

Funding for this research was provided by the U.S. Geological Survey Climate Research and Development Program and by the U.S. Geological Survey Mendenhall Postdoctoral Research Fellowship Program. This research used samples provided by the International Ocean Discovery Program (IODP). This is a product of the U.S. Geological Survey PRISM Project. 


\section{References Cited}

Blow, W.H., 1969, Late middle Eocene to recent planktonic foraminiferal biostratigraphy, in Brönnimann, P., and Renz, H.H., eds., Proceedings of the First International Conference on Planktonic Microfossils: Leiden, Netherlands, E.J. Brill, p. 199-422.

Dowsett, Harry, Robinson, Marci, and Foley, Kevin, 2015, A global planktic foraminifer census data set for the Pliocene ocean: Scientific Data, v. 2, article 150076, 6 p. [Also available at https://doi.org/10.1038/sdata.2015.76.]

Dowsett, H.J., 1991, The development of a long-range foraminifer transfer function and application to late Pleistocene North Atlantic climatic extremes: Paleoceanography, v. 6, no. 2, p. 259-273. [Also available at https://doi.org/10.1029/90PA02541.]

Dowsett, H.J., Foley, K.M., Stoll, D.K., Chandler, M.A., Sohl, L.E., Bentsen, Mats, OttoBliesner, B.L., Bragg, F.J., Chan, W.-L., Contoux, Camille, Dolan, A.M., Haywood, A.M., Jonas, J.A., Jost, Anne, Kamae, Youichi, Lohmann, Gerrit, Lunt, D.J., Nisancioglu, K.H., AbeOuchi, Ayako, Ramstein, Gilles, Riesselman, C.R., Robinson, M.M., Rosenbloom, N.A., Salzmann, Ulrich, Stepanek, Christian, Strother, S.L., Ueda, Hiroaki, Yan, Qing, and Zhang, Zhongshi, 2013, Sea surface temperature of the mid-Piacenzian ocean-A data-model comparison: Scientific Reports, v. 3, 8 p. [Also available at https://doi.org/10.1038/srep02013.]

Dowsett, H.J., and Poore, R.Z., 1990, A new planktic foraminifer transfer function for estimating Pliocene-Holocene paleoceanographic conditions in the North Atlantic: Marine Micropaleontology, v. 16, nos. 1-2, p. 1-23. [Also available at https://doi.org/10.1016/03778398(90)90026-I.]

Dowsett, H.J., and Robinson, M.M., 2007, Mid-Pliocene planktic foraminifer assemblage of the North Atlantic Ocean: Micropaleontology, v. 53, nos. 1-2, p. 105-126. [Also available at https://doi.org/10.2113/gsmicropal.53.1-2.105.]

Dowsett, H.J., Robinson, M.M., and Foley, K.M., 2009, Pliocene three-dimensional global ocean temperature reconstruction: Climate of the Past, v. 5, no. 4, p. 769-783. [Also available at https://doi.org/10.5194/cp-5-769-2009.]

Farrell, J.W., Clemens, S.C., and Gromet, L.P., 1995, Improved chronostratigraphic reference curve of late Neogene seawater ${ }^{87} \mathrm{Sr} /{ }^{86} \mathrm{Sr}$ : Geology, v. 23, no. 5, p. 403-406. [Also available at https://doi.org/10.1130/0091-7613(1995)023<0403:ICRCOL>2.3.CO;2.]

Foley, K., Robinson, M., Dowsett, H.J., Poore, R.Z., Stoll, D., Polanco, E., Wiggs, L., Caballero, R., Verardo, S., Strother, S., West, S., Lutz, B., and Brown, S., 2015, A global planktic foraminifer census data set for the Pliocene ocean: National Climatic Data Center, Paleoclimatology Data web page. [Also available at https://www.ncdc.noaa.gov/paleosearch/study/19281.]

Herbert, T.D., Peterson, L.C., Lawrence, K.T., and Liu, Zhonghui, 2010, Tropical ocean temperatures over the past 3.5 million years: Science, v. 328, no. 5985, p. 1530-1534. [Also available at https://doi.org/10.1126/science.1185435.]

Hutson, W.H., 1980, The Agulhas Current during the late Pleistocene-Analysis of modern faunal analogs: Science, v. 207, no. 4426, p. 64-66. [Also available at https://doi.org/10.1126/science.207.4426.64.] 
Karas, Cyrus, Nürnberg, Dirk, Tiedemann, Ralf, and Garbe-Schönberg, Dieter, 2011, Pliocene Indonesian Throughflow and Leeuwin Current dynamics-Implications for Indian Ocean polar. heat flux: Paleoceanography, v. 26, no. 2, article PA2217, 9 p. [Also available at https://doi.org/10.1029/2010PA001949.]

Kucera, M., and Schönfeld, J., 2007, The origin of modern oceanic foraminiferal faunas and Neogene climate change, in Williams, M., Haywood, A.M., Gregory, F.J., and Schmidt, D.N., eds., Deep-time perspectives on climate change-Marrying the signal from computer models and biological proxies: Micropalaeontological Society Special Publication [2], p. 409-426. [Published by the Geological Society of London.] [Also available at https://doi.org/10.1144/TMS002.18.]

Parker, F.L., 1962, Planktonic foraminiferal species in Pacific sediments: Micropaleontology, v. 8, no. 2, p. 219-254. [Also available at https://doi.org/10.2307/1484745.]

Parker, F.L. 1967, Late Tertiary biostratigraphy (planktonic foraminifera) of tropical IndoPacific deep-sea cores: Bulletins of American Paleontology, v. 52, no. 235, p. 111-208.

Prell, W.L., Martin, A., Cullen, J.L., and Trend, M., 1999, Brown University Foraminiferal Database: IGBP PAGES/World Data Center-A for Paleoclimatology, Data Contribution Series \#1999-027, NOAA/NGDC Paleoclimatology Program. [Also available at ftp://ftp.ncdc.noaa.gov/pub/data/paleo/paleocean/brown_foram/.]

Reynolds, R.W., and Smith, T.M., 1995, A high-resolution global sea surface temperature climatology: Journal of Climate, v. 8, no. 6, p. 1571-1583. [Also available at https://doi.org/10.1175/1520-0442(1995)008<1571:AHRGSS>2.0.CO;2.]

Rio, Domenico; Fornaciari, Eliana; and Raffi, Isabella, 1990, Late Oligocene through early Pleistocene calcareous nannofossils from western equatorial Indian Ocean (leg 115), in Duncan, R.A., Backman, Jan, Dunbar, R.B., and Peterson, L.C., eds., Mascarene Plateau *** sites 705-716 ***: Proceedings of the Ocean Drilling Program, Scientific Results, v. 115, p. 175-235. [Also available at https://doi.org/10.2973/odp.proc.sr.115.152.1990.]

Robinson, M.M., Dowsett, H.J., Dwyer, G.S., and Lawrence, K.T., 2008, Reevaluation of midPliocene North Atlantic sea surface temperatures: Paleoceanography, v. 23, no. 3, article PA3213, 9 p. [Also available at https://doi.org/10.1029/2008PA001608.]

Shipboard Scientific Party, 1989a, Site 754, in Broken Ridge and Ninetyeast Ridge *** sites 752-758 ***: Proceedings of the Ocean Drilling Program, Initial Reports, v. 121, p. 191-236. [Also available at https://doi.org/10.2973/odp.proc.ir.121.108.1989.]

Shipboard Scientific Party, 1989b, Site 757, in Broken Ridge and Ninetyeast Ridge *** sites 752-758 ***: Proceedings of the Ocean Drilling Program, Initial Reports, v. 121, p. 305-358. [Also available at https://doi.org/10.2973/odp.proc.ir.121.111.1989.]

Waterson, A.M., Edgar, K.M., Schmidt, D.N., and Valdes, P.J., 2017, Quantifying the stability of planktic foraminiferal physical niches between the Holocene and Last Glacial Maximum: Paleoceanography, v. 32, no. 1, p. 74-89. [Also available at https://doi.org/10.1002/2016PA002964.] 
ISSN 2331-1258 (online)

https://doi.org/10.3133/ofr20171158 